\title{
Analysis of time trends in preemptive kidney transplantation and effect of pre-transplant dialysis duration on graft survival: a nationwide cohort study
}

\author{
Jeong-Hoon Lim¹, Sang-Ho Lee ${ }^{2}$, Yu Ho Lee ${ }^{3}$, Jung Pyo Lee ${ }^{4}$, Jaeseok Yang ${ }^{5}$, Myoung Soo Kim ${ }^{6}$, Sun-Hee Park ${ }^{1}$, \\ Chan-Duck Kim ${ }^{1}$, Jang-Hee Cho ${ }^{1}$

\footnotetext{
${ }^{1}$ Division of Nephrology, Department of Internal Medicine, School of Medicine, Kyungpook National University, Daegu, Korea

${ }^{2}$ Division of Nephrology, Department of Internal Medicine, Kyung Hee University Hospital at Gangdong, Seoul, Korea

${ }^{3}$ Division of Nephrology, Department of Internal Medicine, Bundang CHA General Hospital, Seongnam, Korea

${ }^{4}$ Division of Nephrology, Department of Internal Medicine, Seoul National University College of Medicine, Seoul, Korea

${ }^{5}$ Department of Surgery, Seoul National University Hospital, Seoul, Korea

${ }^{6}$ Department of Surgery, Yonsei University College of Medicine, Seoul, Korea
}

Background: Preemptive kidney transplantation (KT) has advantages on graft survival, quality of life, and medical expense. However, the trend of preemptive KT over time is not known. This study analyzed the time trend of preemptive KT and the effect of pre-transplant dialysis duration on post-transplant outcomes.

Methods: Using a nationwide cohort study data from 2014 to 2019, a total of 3,392 living donor KT (LDKT) patients were enrolled. The annual proportion of preemptive KT was examined by a trend analysis. Factors associated with preemptive KT were analyzed using multivariate logistic regression. Graft survival was compared using Cox proportional hazards regression according to pre-transplant dialysis duration.

Results: Preemptive KT was 816 (24.1\%) among LDKT and mean estimated glomerular filtration rate was $8.0 \mathrm{mg} / \mathrm{dL} / 1.73 \mathrm{~m}^{2}$ at the time of transplant. Transplantation year was independently associated with preemptive KT (adjusted odds ratio [aOR], 0.95; 95\% confidence interval [Cl], 0.89-1.00; $\mathrm{P}=0.046)$. KT from glomerulonephritis was a predictor of preemptive $\mathrm{KT}$ (aOR, 1.41; 95\% Cl, 1.14-1.76; $\mathrm{P}=0.002)$; however, desensitization was associated with non-preemptive $\mathrm{KT}$ (aOR, 0.67; 95\% $\mathrm{Cl}, 0.56-0.80$; $\mathrm{P}<0.001)$. The annual trend analysis revealed that preemptive KT gradually decreased over time; diabetes increased whereas glomerulonephritis decreased among the underlying causes of preemptive KT (all $\mathrm{P}<0.05)$. Patients with dialysis longer than 6 months before KT showed an increased risk of graft failure than preemptive KT (adjusted hazard ratio, 2.54; 95\% Cl, 1.10-5.88; $\mathrm{P}=0.029)$; however, pre-transplant dialysis less than 6 months showed comparable graft survival with preemptive KT.

Conclusions: Preemptive KT is declining every year, associated with an increase in diabetes and a decrease in glomerulonephritis as underlying cause of KT. Short period of dialysis less than 6 months does not affect graft survival compared to preemptive $\mathrm{KT}$; however, dialysis longer than 6 months decreases graft survival.

Corresponding author: Jang-Hee Cho

E-mail: jh-cho@knu.ac.kr

(c) The Korean Society for Transplantation

This is an Open Access article distributed under the terms of the Creative Commons Attribution Non-Commercial License (http://creativecommons.org/licenses/by-nc/4.0/) which permits unrestricted non-commercial use, distribution, and reproduction in any medium, provided the original work is properly cited. 